\title{
ARROWHEAD OPERATORS ON A HILBERT SPACE
}

\author{
J. Abderramán MarRero AND V. TOMEO
}

\begin{abstract}
The arrowhead matrices define a class of one-term Sylvester matrix (OTSM) operators on a finite-dimensional Hilbert space through an elementary $U D L$ factorization. It enables us to consider the infinite invertible arrowhead matrices $U D L$ factored properly for introducing, under suitable conditions, the arrowhead operators and their associated class of OTSM operators on an infinite-dimensional Hilbert space. Properties regarding convergence, inertia, inverses, and spectra are also considered.
\end{abstract}

Mathematics subject classification (2010): 15A99, 47B99.

Keywords and phrases: Infinite arrowhead matrix, matrix factorization, arrowhead operator, one-term Sylvester matrix operator.

\section{REFERENCES}

[1] J. Abderramán Marrero, V. Tomeo, E. Torrano, On inverses of infinite Hessenberg matrices, J. Comp. Appl. Math. 275 (2015) 356-365.

[2] N. I. AKhIEZER, I. M. GlaZMAn, Theory of linear operators in Hilbert space, Dover Publications Inc, New York, USA 1993.

[3] M. Bixon, J. Jortner, Intramolecular radiationless transitions, J. Chem. Phys. 48 (1968) 715-726.

[4] J. W. BunCE, Inertia and controllability in infinite dimensions, J. Math. Anal. Appl. 129 (1988) 569580.

[5] B. E. CAIn, An inertia theory for operators on a Hilbert space, J. Math. Anal. Appl. 41 (1973) $97-$ 114.

[6] R. G. Cooke, Infinite matrices \& sequence spaces, Dover Publications, New York, USA 1955.

[7] J. M. GADZUK, Localized vibrational modes in Fermi liquids, general theory, Phys. Rev. B. 24 (1981) $1651-1663$.

[8] I. Gohberg, S. Goldberg, M. A. KAAShoek, Basic classes of linear operators, Birkhäuser Verlag, Basel, Switzerland, 2003.

[9] T. Kato, Perturbation theory for linear operators, 2nd edition, Springer-Verlag, Berlin, Germany, 1982.

[10] M. Konstantinov, V. Mehrmann, P. Petkov, On properties of Sylvester and Lyapunov operators, Linear Algebra Appl. 312 (2000) 35-71.

[11] E. KREYSZIG, Introductory functional analysis with applications, John Wiley \& Sons, New York, USA 1989.

[12] H. T. KUng, B. W. SUTER, A hub matrix theory and applications to wireless communications, EURASIP J. Adv. Signal Process. (2007) Article ID 136598 pages.

[13] P. LAnCASter, M. Tismenetsky, The theory of matrices, 2nd edition. Academic Press, San Diego, CA, USA 1985.

[14] B. N. PARLETt, The symmetric eigenvalue problem, SIAM, Philadelphia, USA 1998.

[15] D. P. O'LeARY, G. W. STEWART, Computing the eigenvalues and eigenvectors of symmetric arrowhead matrices, J. Comput. Phys. 90 (1990) 497-505.

[16] A. Ostrows Ki, H. Schneider, Some theorems on the inertia of general matrices, J. Math. Anal. Appl. 4 (1962) 72-84.

[17] P. N. Shivakumar, K. C. Sivakumar, A review of infinite matrices and their applications, Linear Algebra Appl. 430 (2009) 976-998. 
[18] J. H. WILKInSON, The algebraic eigenvalue problem, Oxford University Press, New York, USA 1965. 